P3.128 DEVELOPMENT OF A RISK CALCULATOR FOR THE 3MONTH PREDICTION OF INCIDENT SYPHILIS INFECTION AMONG HIGH-RISK MEN WHO HAVE SEX WITH MEN AND TRANSGENDER WOMEN PRESENTING TO A STD CLINIC IN LIMA, PERU

${ }^{1}$ Lao-Tzu Allan-Blitz, ${ }^{2}$ Kelika A Konda, ${ }^{3}$ Silver K Vargas, ${ }^{3}$ Eddy R Segura, ${ }^{3}$ Gino M Calvo, ${ }^{3}$ Carlos F Caceres, ${ }^{2}$ Jeffrey D Klausner. ${ }^{1}$ David Geffen School of Medicine, UCLA, And Saphir, Los Angeles, USA; 'Department of Medicine, David Geffen School of Medicine, University of California Los Angeles Los Angeles, USA; ${ }^{3}$ Universidad Peruana Cayetano Heredia, Lima - Peru

10.1136/sextrans-2017-053264.363

Introduction Syphilis incidence worldwide has rebounded since 2000, especially among men who have sex with men (MSM). A predictive model for incident syphilis infection may inform prevention counselling and use of chemoprophylaxis.

Methods We analysed data from a longitudinal study of a STD clinic-based cohort of MSM and transgender women reporting a history of HIV or syphilis infection and/or highrisk sexual behaviour, followed quarterly for two years. Incident infection was defined as a four-fold increase in RPR titers or new RPR reactivity if two prior titers were non-reactive. We used generalised estimating equations with a Poisson regression to develop a predictive model of syphilis incidence in one-half of the data set, and verified the model in the second half, calculating an area under the curve (AUC), summarising specificity and sensitivity. We then applied the final model to the full baseline dataset. Finally we developed an online risk calculator from our model.

Results Among 401 participants enrolled, 22\% were transgender women and $31 \%$ were HIV-infected at baseline. Syphilis incidence was 19.9 cases per 100-person years (95\% CI 16.324.3). Predictors of syphilis incidence were HIV infection, high number of male sex partners (categorised as: $0,1,2-3$, $4-9,>10)$, history of syphilis infection, receptive and versatile anal sex role and condomless receptive anal sex. The AUC was $71 \%(95 \%$ CI $64 \%-78 \%)$ in the validation dataset for incident syphilis infection in the next 3 months. Those at highest risk had a 1-in-7 likelihood of syphilis infection in the next 3 months. When applied to the baseline dataset the AUC was $71 \%$ (95\% CI 65\%-77\%) for predicting recent syphilis infection. The online syphilis risk calculator is available at: www.syphrisk.net (English), www.sifriesgo.net (Spanish).

Conclusion Our results show that the likelihood of syphilis infection among a high-risk STD clinic-based cohort can be estimated accurately. Our calculator may guide STD clinical management by directing risk behaviour counselling and potential use of doxycycline chemoprophylaxis.

\section{P3.129 HOW CAN WE USE PHYLOGENETICS TO FACILITATE CLINICAL CASE FINDING AND PARTNER NOTIFICATION IN HIV: LESSONS FROM A SYSTEMATIC REVIEW OF ITS USE IN STIGMATISED INFECTIOUS DISEASES}

${ }^{1}$ Larissa Mulka, 'Jaime H Vera, ${ }^{2}$ Andrew J Leigh-Brown, ${ }^{1}$ Jackie A Cassell. ${ }^{1}$ Brighton and Sussex Medical School, Brighton, UK; ${ }^{2}$ University of Edinburgh, Edinburgh, UK

\subsection{6/sextrans-2017-053264.364}

Introduction Phylogenetic information provides new horizons for clinical case finding in HIV, but raises issues of acceptability, privacy and even criminalisation. We reviewed studies describing use of phylogenetics to directly inform case finding in community acquired stigmatised infectious diseases.

Methods A search in MEDLINE, Embase, CINAHL and PsychINFO for articles where phylogenetics have been used to facilitate case finding in sexually transmitted infections, TB, HBV or HCV, published until July 2016 in English.

Results 26 of 6042 papers screened met the inclusion criteria; 17 TB, 9 HIV. 19 studies reported using phylogenetics to identify and investigate HIV outbreaks but did not report its role in case finding. Case finding strategies included confirming the source of an outbreak to prompt wider investigation (HIV); investigation of phylogenetically clustered cases (TB, HIV); combined cluster and geographical information to target screening (TB); screening informed by discrepancies between genotypic and epidemiological data (TB); phylogenetic characterisation to inform a screening intervention (HIV); epidemiological data to identify of a source (HIV); and contact tracing with genotype matching to a phenotype (HIV). Facilitators included sharing molecular surveillance data to establish community support in targeted TB screening. Barriers included delayed results, time lapse between cases and refusal of access to premises for screening. However patient barriers were rarely reported. Ethical issues included media coverage of an HIV sources identity.

Conclusion Phylogenetics-informed approaches to case finding are feasible in stigmatised infections. However studies reporting their use in clinical and public health practice provide limited information on patient related barriers, acceptability, or on ethical challenges such as identification of "core" transmitters or criminalisation. Research into patient views on acceptability, risks and preferred approaches to using phylogenetic information for case finding in HIV is needed to inform interventions.

\section{P3.130 POTENTIAL IMPACT OF TESTING FOR MYCOPLASMA GENITALIUM INFECTION AND MACROLIDE RESISTANCE: A MATHEMATICAL MODELLING ANALYSIS}

${ }^{1}$ Laura C Chambers, ${ }^{2}$ Lisa E Manhart, ${ }^{1}$ Rachel A Silverman, ${ }^{3}$ Ruanne V Barnabas. ${ }^{1}$ University of Washington, Department of Epidemiology, Seattle, USA; ${ }^{2}$ University of Washington, Departments of Epidemiology and Global Health, Seattle, USA; ${ }^{3}$ University of Washington, Departments of Medicine, Global Health, and Epidemiology, Seattle, USA

\subsection{6/sextrans-2017-053264.365}

Introduction Patients with genitourinary symptoms are generally treated syndromically with azithromycin, which can induce macrolide resistance in Mycoplasma genitalium (MG). Directing treatment based on aetiology and known macrolide susceptibility may prevent emergence of resistance. We constructed a mathematical model to evaluate the potential impact of simultaneous detection of $\mathrm{MG}$ and resistance markers on the percent of MG infections that are macrolidesusceptible.

Methods We developed a gender- and risk-stratified, compartmental model of MG transmission within a heterosexual population. We assumed clearance of untreated infections in 30 days; development of symptoms in $2.4 \%$ of infected men and $5.1 \%$ of infected women; initial treatment of symptomatic men and women with azithromycin; treatment of men with persistent/recurrent symptoms with moxifloxacin; 50\% of infections macrolide-susceptible at baseline; de-novo macrolide resistance in $18 \%$ of susceptible bacteria after azithromycin therapy; and 100\% efficacy of moxifloxacin. The model was 